\title{
Thoracoscopic lobectomy following neoadjuvant tyrosine kinase inhibitor treatment
}

\author{
Neel P. Chudgar, MD, and David R. Jones, MD, New York, NY
}

\footnotetext{
From the Thoracic Service, Department of Surgery, Memorial Sloan Kettering Cancer Center, New York, NY. Supported, in part, by a National Institutes of Health/National Cancer Institute Cancer Center Support Grant (No. P30 CA008748)

Disclosures: Dr Jones has served in consultant and advisory roles for AstraZeneca and Merck. Dr Chudgar reported no conflicts of interest.

The Journal policy requires editors and reviewers to disclose conflicts of interest and to decline handling or reviewing manuscripts for which they may have a conflict of interest. The editors and reviewers of this article have no conflicts of interest.

Received for publication Feb 2, 2021; accepted for publication Feb 2, 2021; available ahead of print Feb 10, 2021. Address for reprints: David R. Jones, MD, Thoracic Service, Department of Surgery, Memorial Sloan Kettering Cancer Center, 1275 York Ave, Box 7, New York, NY 10065 (E-mail: jonesd2@mskcc.org).

JTCVS Techniques 2021;7:294-7

2666-2507

Copyright (C) 2021 The Authors. Published by Elsevier Inc. on behalf of The American Association for Thoracic Surgery. This is an open access article under the CC BY-NC-ND license (http://creativecommons.org/licenses/bync-nd/4.0/).

https://doi.org/10.1016/j.xjtc.2021.02.005
}

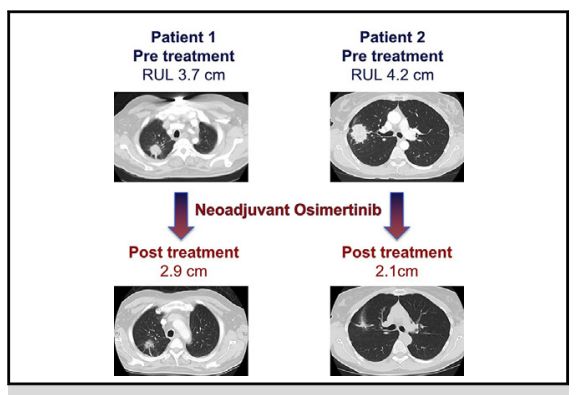

Pre- and postinduction imaging of 2 patients treated with neoadjuvant osimertinib.

\begin{abstract}
CENTRAL MESSAGE
Degree of fibrosis, preservation of tissue planes, technical difficulty, and operative duration were not predictable preoperatively in 2 patients undergoing thoracoscopic lobectomy after TKI therapy.
\end{abstract}

See Commentaries on pages 298 and 299.
Tyrosine kinase inhibitor (TKI) therapy is approved for the treatment of stage IV non-small cell lung cancer (NSCLC) harboring an activating epidermal growth factor receptor (EGFR) mutation. Comparison of TKI versus conventional platinum doublet-based chemotherapy in stage IV NSCLC has demonstrated improved progression-free survival with TKI therapy. ${ }^{1,2}$ The recently published phase III FLAURA trial found superior overall survival with osimertinib, a third-generation TKI, compared with the secondgeneration TKIs gefitinib or erlotinib, in patients with previously untreated EGFR mutation-positive advanced NSCLC. ${ }^{3}$ These findings resulted in Food and Drug Administration approval of osimertinib as a first-line therapy for locoregional or metastatic NSCLC. The recent ADAURA trial demonstrated significantly improved disease-free survival with adjuvant osimertinib for completely resected stage IB to IIIA EGFR-mutant NSCLC. ${ }^{4}$

Notwithstanding these seminal publications, limited data exist on the surgical aspects of lung cancer resection after neoadjuvant TKI therapy. Specifically, reports on the degrees of tissue fibrosis and difficulty in dissection are not currently available, which may become more relevant as an increasing number of patients are treated preoperatively with targeted therapy. We present 2 patients with locally advanced EGFR-mutant NSCLC treated with neoadjuvant osimertinib followed by thoracoscopic lobectomy and complete lymphadenectomy. Both patients received care in a multidisciplinary setting in consultation with both thoracic surgery and medical oncology. Written informed consent was obtained for publication of this report.

\section{CASE PRESENTATIONS}

Patient 1, a 78-year-old man with a light smoking history, presented with a cough and was found to have a right upper lobe mass. Biopsy demonstrated adenocarcinoma with an exon 21 EGFR (L858R) point mutation. Mediastinal evaluation via endobronchial ultrasound-guided fine-needle aspiration demonstrated metastatic adenocarcinoma in a station 7 lymph node. The patient was clinically staged as $\mathrm{cT}_{2 \mathrm{a}} \mathrm{N}_{2}$ $\mathrm{M}_{0}$. Induction chemotherapy was considered, but following identification of the activating EGFR mutation and multidisciplinary consultation, the patient agreed to off-protocol TKI therapy. He received 3 months of osimertinib $(80 \mathrm{mg}$ daily), which was tolerated without toxicity. Positronemission tomography (PET) imaging demonstrated a partial 


\section{Patient 1}

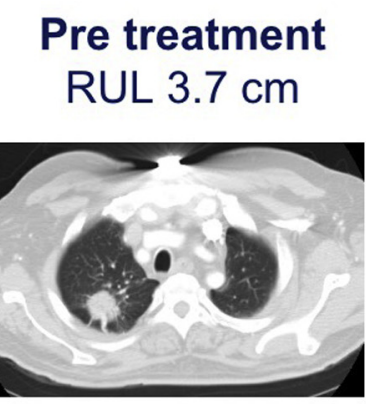

$\begin{array}{ccc}\text { Tumor } & \text { R hilar LN } & \text { Subcarinal LN } \\ \text { SUV 6.4 } & \text { SUV } 10.0 & \text { SUV } 4.9\end{array}$

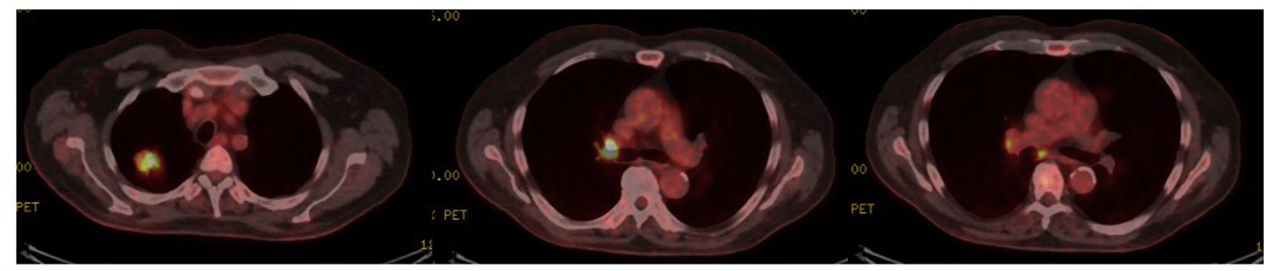

\section{Post treatment}

$2.9 \mathrm{~cm}$

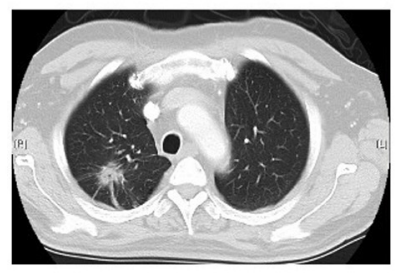

Pre treatment

RUL $4.2 \mathrm{~cm}$

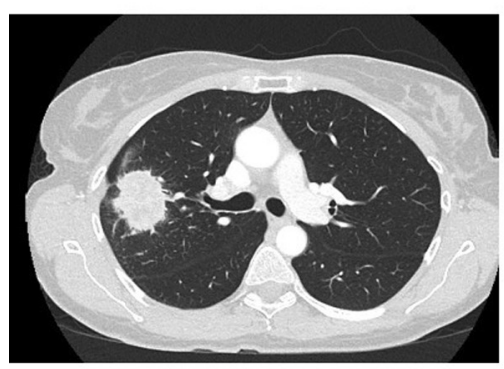

Post treatment

$2.1 \mathrm{~cm}$

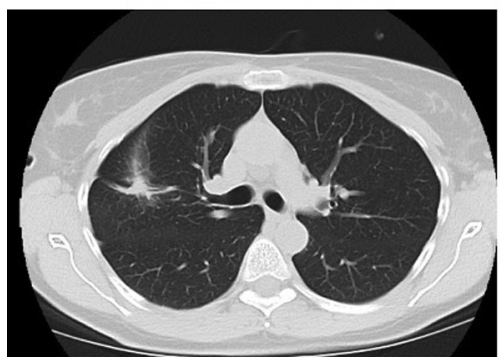

SUV 2.2

Non-avid

Non-avid

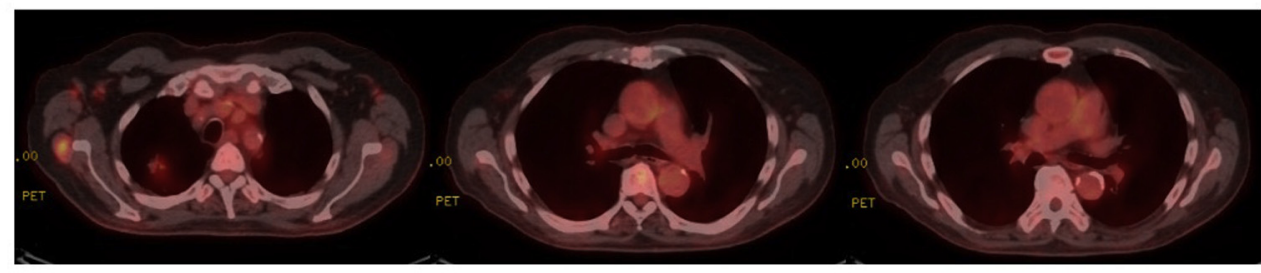

\section{Patient 2}

\section{Tumor}

SUV 13.9

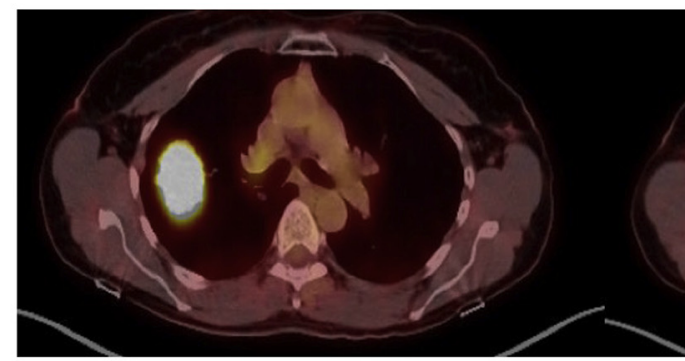

R hilar LN

SUV 4.0

\section{SUV 1.3}

Non-avid

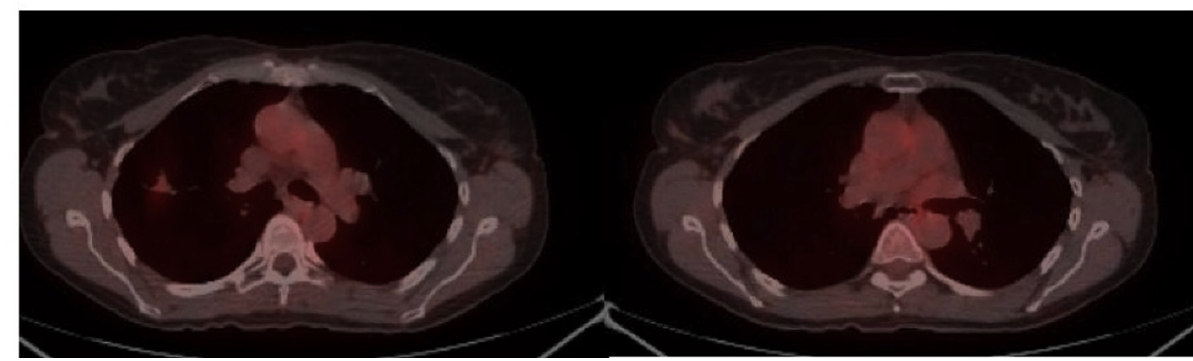

FIGURE 1. Pre- and posttreatment imaging, demonstrating response to therapy in both patients. RUL, Right upper lobe; $S U V$, standardized uptake value; $L N$, lymph node. 


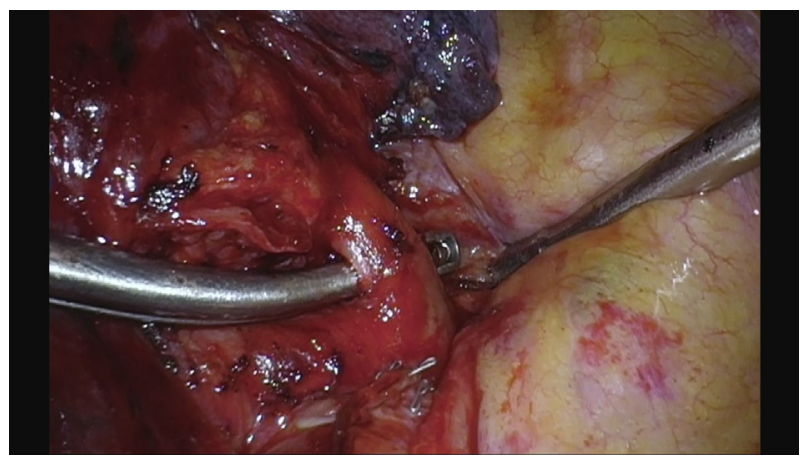

VIDEO 1. Thoracoscopic right upper lobectomy performed on patient 1, demonstrating a technically challenging lymphadenectomy. Video available at: https://www.jtcvs.org/article/S2666-2507(21)00133-4/fulltext.

radiographic response with a decrease in the size of the lesion from 3.7 to $2.9 \mathrm{~cm}$, reduction in the maximum standardized uptake value of the primary tumor ( 6.4 vs 2.2 ), and reduced avidity in station 7 nodes (Figure 1). The patient underwent video-assisted thoracoscopic (VATS) right upper lobectomy (Video 1). Extensive fibrotic changes were found on subcarinal and hilar dissection, which increased the operative duration (total, 170 minutes). Tissue planes were difficult to ascertain, particularly when dissecting the upper lobe vessels. Dissection of the truncus anterior was tedious and required sharp dissection for safe and adequate exposure before staple ligation. VATS lobectomy was ultimately completed without complication, and the patient was discharged on postoperative day 3 . Final pathologic assessment demonstrated a yp $\mathrm{T}_{1 \mathrm{~b}} \mathrm{~N}_{2} \mathrm{M}_{0} 2.5$-cm tumor with $60 \%$ tumor viability. Station 7 nodes were pathologically negative; however, the level $2 \mathrm{R}$ node contained residual malignancy. The patient received adjuvant cisplatin and pemetrexed and

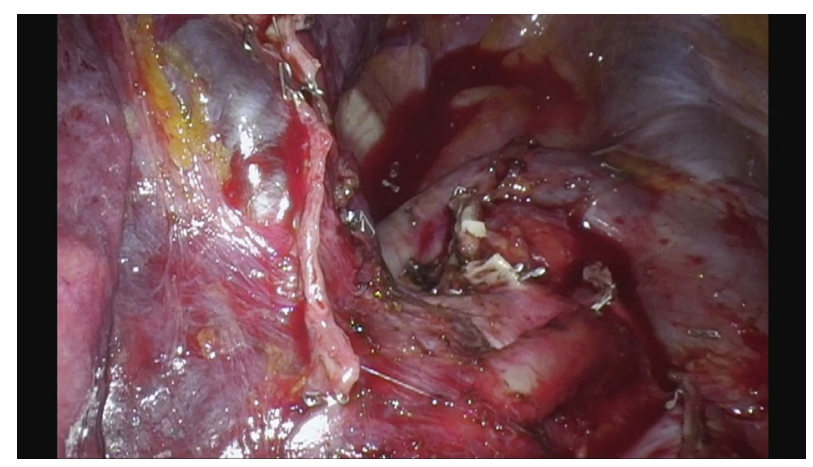

VIDEO 2. Thoracoscopic right upper lobectomy performed on patient 2, demonstrating an uncomplicated resection. Video available at: https:// www.jtcvs.org/article/S2666-2507(21)00133-4/fulltext.

remains without evidence of disease 16 months postoperatively; he has not continued osimertinib.

Patient 2, a 60-year-old woman who had never smoked, presented with a right upper lobe mass and hilar adenopathy. Biopsy demonstrated adenocarcinoma with an EGFR exon 19 deletion. Although endobronchial ultrasound-guided biopsies were negative at $4 \mathrm{R}$ and $10 \mathrm{R}$, the patient's disease was clinically staged as $T_{2 b} N_{1} M_{0}$ based on computed tomography-PET imaging. Following multidisciplinary consultation and informed consent, she received neoadjuvant osimertinib ( $80 \mathrm{mg}$ daily) for 7 months on the basis of treatment to a best-radiographic-response protocol. She experienced grade I fatigue and diarrhea while receiving treatment. Follow-up PET imaging demonstrated a decrease in tumor size from 4.2 to $2.1 \mathrm{~cm}$, a robust decrease in tumor maximum standardized uptake value (13.9 vs 1.3), and elimination of avidity in the right hilum (Figure 1). VATS right upper lobectomy was performed (Video 2). Nodal dissection was less tedious than in the prior case, allowing for the

TABLE 1. Clinicopathologic characteristics and outcomes of the 2 patients who underwent lobectomy after osimertinib treatment

\begin{tabular}{|c|c|c|}
\hline Variable & Patient 1 & Patient 2 \\
\hline Age & 78 & 60 \\
\hline Sex & Male & Female \\
\hline Mutation status & Exon $21 \mathrm{~L} 858 \mathrm{R}$ & Exon 19 deletion \\
\hline Clinical staging & $\mathrm{cT}_{2 \mathrm{a}} \mathrm{N}_{2} \mathrm{M}_{0}$ & $\mathrm{cT}_{2 \mathrm{~b}} \mathrm{~N}_{1} \mathrm{M}_{0}$ \\
\hline Smoking history & $<5$ pack-years & Never-smoker \\
\hline Duration of TKI treatment (mo) & 3 & 7 \\
\hline TKI toxicity & None & Grade 1 diarrhea, fatigue \\
\hline Operative time (min) & 170 & 141 \\
\hline Estimated blood loss (mL) & 50 & 25 \\
\hline Pathologic staging & $\operatorname{ypT}_{1 b} N_{2} M_{0}$ & $\mathrm{ypT}_{1 \mathrm{a}} \mathrm{N}_{0} \mathrm{M}_{0}$ \\
\hline Histologic subtype: Acinar (\%) & 70 & 100 \\
\hline Viable tumor $(\%)$ & 60 & 10 \\
\hline Adjuvant therapy & 4 Cycles cisplatin and pemetrexed & 4 Cycles cisplatin and pemetrexed + osimertinib for $3 y$ \\
\hline
\end{tabular}

TKI, Tyrosine kinase inhibitor. 
procedure to be completed in 141 minutes. Even with a longer duration of osimertinib therapy, the tissue planes were preserved, with less fibrosis. Final pathology demonstrated a $\operatorname{ypT}_{1 \mathrm{a}} \mathrm{N}_{0} \quad \mathrm{M}_{0} 3.3 \mathrm{-cm}$ tumor with $10 \%$ tumor viability. The patient was discharged on postoperative day 2 without complication. She received adjuvant cisplatin and pemetrexed and will continue osimertinib for 3 years postoperatively (Table 1).

\section{DISCUSSION}

At present, there are at least 7 actionable genomic alterations with targeted therapies available for patients with NSCLC. Chemotherapy with or without radiation has been the standard neoadjuvant therapy for the past 25 years. Recently, immune checkpoint inhibitors alone or combined with chemotherapy have been associated with substantial tumor responses. ${ }^{5,6}$ Combined immunotherapy regimens allow for performance of minimally invasively procedures, but may result in more desmoplastic and fibrotic responses, which may lead to higher rates of conversion to open thoracotomy. ${ }^{7}$

These 2 cases of induction osimertinib followed by VATS lobectomy demonstrate the challenges in predicting the degree of postinduction fibrosis, nodal flare related to reactive or inflammatory changes, and difficulty of dissection. With the expanding use of targeted therapies, their addition to neoadjuvant treatment paradigms is being explored. The phase III international NeoADAURA trial, examining neoadjuvant osimertinib for $E G F R$-mutant tumors, is open to accrual. Given the strong preference by thoracic surgical oncologists to perform minimally invasive resections, it is expected that more of these procedures will be performed successfully, even after TKI therapy.

\section{CONCLUSIONS}

We present 2 cases demonstrating the successful use of VATS following induction TKI therapy, while simultaneously highlighting the variable degrees of fibrosis, loss of tissue planes, and operative time.

\section{References}

1. Mok TS, Wu YL, Thongprasert S, Yang CH, Chu DT, Saijo N, et al. Gefitinib or carboplatin-paclitaxel in pulmonary adenocarcinoma. N Engl J Med. 2009;361: 947-57.

2. Rosell R, Carcereny E, Gervais R, Vergnenegre A, Massuti B, Felip E, et al. Erlotinib versus standard chemotherapy as first-line treatment for European patients with advanced EGFR mutation-positive non-small-cell lung cancer (EURTAC): a multicentre, open-label, randomised phase 3 trial. Lancet Oncol. 2012;13: 239-46.

3. Soria JC, Ohe Y, Vansteenkiste J, Reungwetwattana T, Chewaskulyong B, Lee KH, et al. Osimertinib in untreated EGFR-mutated advanced non-small-cell lung cancer. $N$ Engl J Med. 2018;378:113-25.

4. Wu YL, Tsuboi M, He J, John T, Grohe C, Majem M, et al. Osimertinib in resected EGFR-mutated non-small-cell lung cancer. N Engl J Med. 2020;383:1711-3.

5. Forde PM, Chaft JE, Smith KN, Anagnostou V, Cottrell TR, Hellmann MD, et al Neoadjuvant PD-1 blockade in resectable lung cancer. N Engl J Med. 2018;378 1976-86.

6. Provencio M, Nadal E, Insa A, García-Campelo MR, Casal-Rubio J, Dómine M, et al. Neoadjuvant chemotherapy and nivolumab in resectable non-small-cell lung cancer (NADIM): an open-label, multicentre, single-arm, phase 2 trial. Lancet Oncol. 2020;21:1413-22.

7. Stiles BM, Sepesi B, Broderick SR, Bott MJ. Perioperative considerations for neoadjuvant immunotherapy in non-small cell lung cancer. J Thorac Cardiovasc Surg. 2020;160:1376-82. 